\title{
A Theorem on Numbers of the Form $10^{x}$
}

\author{
Ravin Kumar ${ }^{\text {[0000-0002-3416-2679] }}$ \\ Department of Computer Science, Meerut Institute of Engineering and Technology, \\ meerut-250005, Uttar Pradesh, INDIA. \\ ravin.kumar.cs.2013@miet.ac.in
}

\begin{abstract}
Number theory is one of the core branches of pure mathematics. It has played an important role in the study of natural numbers. In this paper, we are presenting a theorem on the numbers of form $10^{\mathrm{x}}$, where $\mathrm{x} \in \mathbb{Z}^{+}$. The proposed theorem have a major application in computer science. It can be used to predict ' $\mathrm{n}$ ' bits which will always represent more than $10^{\mathrm{X}}$ total numbers. We proved that the nature of the ' $n$ ' bits is always one of the forms $10 \mathrm{i}, 10 \mathrm{i}+$ 4 , or $10 \mathrm{i}+7$, where $\mathrm{i} \in \mathbf{W}$.
\end{abstract}

Keywords: Number theory, Binary Number System, Modular Arithmetic, $10^{\mathrm{x}}$

\section{Introduction}

Number theory is one of the oldest fields of pure mathematics. It covers board topics dealing with theories focused on subsets of real numbers such as positive integers, rational numbers, and natural numbers [1]. Number theory also deals with the diverse subtopics of modular arithmetic [2], and prime numbers [3].

In this paper, along with the mathematical proof, an application of our proposed theorem is also discussed in computer science.

\section{Proposed Theorem}

For any number of the form $10^{\mathrm{x}}$, where $\mathrm{x} \in \mathbb{Z}^{+}$the following mathematical expression is always true.

$$
2^{\left(\left(10\left\lfloor\frac{x}{3}\right\rfloor\right)+4((x \bmod 3) \bmod 2)+7\left\lfloor\frac{(x \bmod 3)}{2}\right\rfloor\right)}>10^{x}, \text { where } \mathrm{x} \in \mathbb{Z}^{+} .
$$

\subsection{Mathematical Proof}

In a decimal number system, we know that-

$$
\begin{aligned}
& 2^{10}>10^{3} \\
& 2^{4}>10 \\
& 2^{7}>10^{2}
\end{aligned}
$$


From equation 1, 2 and 3, it can be concluded that,

$$
\begin{aligned}
& 2^{10 i}>10^{3 i} \text { where } \mathrm{i} \in \mathbb{Z}^{+} \\
& 2^{4 j} \geq 10^{j} \text { where } \mathrm{j} \in\{0,1\} \\
& 2^{7 k} \geq 10^{2 k} \text { where } \mathrm{k} \in\{0,1\}
\end{aligned}
$$

On combining equations 4, 5 and 6, a new combined form is obtained.

$$
2^{10 i+4 j+7 k}>10^{3 i+j+2 k}
$$

Since, $0 \leq(x \bmod 3) \leq 2$, it can be said that-

$$
\begin{aligned}
& 0 \leq((x \bmod 3) \bmod 2) \leq 1 \text {, where } \mathrm{x} \in \mathbb{Z}^{+} \\
& 0 \leq\left\lfloor\frac{(x \bmod 3)}{2}\right\rfloor \leq 1 \text {, where } \mathrm{x} \in \mathbb{Z}^{+}
\end{aligned}
$$

In equation 7 , we substitute $i$ with $\left\lfloor\frac{x}{3}\right\rfloor, j$ is substituted with $((x \bmod 3) \bmod 2)$ using equation 8 , and $k$ is substituted with $\left\lfloor\frac{(x \bmod 3)}{2}\right\rfloor$ using equation 9. After performing all these substitutions, following equation is obtained.

$2^{\left(\left(10\left\lfloor\frac{x}{3}\right\rfloor\right)+4((x \bmod 3) \bmod 2)+7\left\lfloor\frac{(x \bmod 3)}{2}\right\rfloor\right)}>10^{\left(\left(3\left\lfloor\frac{x}{3}\right\rfloor\right)+((x \bmod 3) \bmod 2)+2\left\lfloor\frac{(x \bmod 3)}{2}\right\rfloor\right)}, \mathrm{x} \in \mathbb{Z}^{+}$

Equation 10 can further be simplified using a well-known relationship [4] of modular arithmetic.

$$
x \bmod y=x-y\left\lfloor\frac{x}{y}\right\rfloor
$$

RHS of equation 10 can further be simplified using equation 11.

$$
\begin{array}{r}
3\left\lfloor\frac{x}{3}\right\rfloor=x-(x \bmod 3) \\
2\left\lfloor\frac{(x \bmod 3)}{2}\right\rfloor=(x \bmod 3)-((x \bmod 3) \bmod 2)
\end{array}
$$

Now simplifying equation 10, using equations 12 and 13.

$$
2^{\left(\left(10\left\lfloor\frac{x}{3}\right\rfloor\right)+4((x \bmod 3) \bmod 2)+7\left\lfloor\frac{(x \bmod 3)}{2}\right\rfloor\right)}>10^{(x-(x \bmod 3)+((x \bmod 3) \bmod 2)+(x \bmod 3)-((x \bmod 3) \bmod 2))}
$$


i.e.

$$
2^{\left(\left(10\left\lfloor\frac{x}{3}\right\rfloor\right)+4((x \bmod 3) \bmod 2)+7\left\lfloor\frac{(x \bmod 3)}{2}\right\rfloor\right)}>10^{x}, \text { where } \mathrm{x} \in \mathbb{Z}^{+}
$$

This proves that our proposed theorem on numbers of the form $10^{\mathrm{x}}$ is mathematically correct.

\section{Applications in Computer Science}

In digital computers, our proposed theorem proves that ' $n$ ' bits of the form $10 \mathrm{i}, 10 \mathrm{i}+$ 4 , or $10 \mathrm{i}+7$, where $\mathrm{i} \in \mathbf{W}$ i.e. whole numbers can always represent numbers greater than $10^{x}$.

$$
2^{n}>10^{x} \text {, where } \mathrm{x} \in \mathbb{Z}^{+}
$$

In equation 15, for a given value of $\mathrm{x}$, value of $\mathrm{n}$ bits can be found using the proposed theorem presented in equation 14.

$$
n=\left(\left(10\left\lfloor\frac{x}{3}\right\rfloor\right)+4((x \bmod 3) \bmod 2)+7\left\lfloor\frac{(x \bmod 3)}{2}\right\rfloor\right) \text {, where } \mathrm{x} \in \mathbb{Z}^{+}
$$

It showed that in a digital computer, for a given $\mathrm{x}$, " $\mathrm{n}$ " bits will always be able to represent a number greater than $10^{x}$, where $\mathrm{x} \in \mathbb{Z}^{+}$. For ease of implementation, a computer program of the proposed theorem is provided open source in the github repository [5].

\section{References}

1. Kraft J, Washington L. An introduction to number theory with cryptography. Chapman and Hall/CRC; 2018 Jan 29.

2. Inam I, Büyükaşık E, editors. Notes from the International Autumn School on Computational Number Theory. Springer International Publishing; 2019.

3. Flath DE. Introduction to number theory. American Mathematical Soc.; 2018 Sep 27.

4. Meidânis J. Lower bounds for arithmetic problems. University of Wisconsin-Madison Department of Computer Sciences; 1990.

5. Github repository, https://github.com/mr-ravin/theorem10, last accessed 2019/7/31. 